\title{
A computer-assisted existence proof for Emden's equation on an unbounded L-shaped domain
}

\author{
Filomena Pacella, Michael Plum and Dagmar Rütters
}

\begin{abstract}
We prove existence, non-degeneracy, and exponential decay at infinity of a non-trivial solution to Emden's equation $-\Delta u=|u|^{3}$ on an unbounded $L$-shaped domain, subject to Dirichlet boundary conditions. Besides the direct value of this result, we also regard this solution as a building block for solutions on expanding bounded domains with corners, to be established in future work. Our proof makes heavy use of computer assistance: Starting from a numerical approximate solution, we use a fixed-point argument to prove existence of a near-by exact solution. The eigenvalue bounds established in the course of this proof also imply non-degeneracy of the solution.
\end{abstract}

\section{Introduction}

In this paper we are concerned with the existence of non-trivial solutions of the problem

$$
\left\{\begin{aligned}
-\Delta u & =|u|^{3} & & \text { in } \Omega \\
u & =0 & & \text { on } \partial \Omega
\end{aligned}\right.
$$

in a planar $L$-shaped unbounded domain

$$
\Omega=((-1, \infty) \times(0,1)) \cup((-1,0) \times(-\infty, 1)) \subset \mathbb{R}^{2} .
$$

It is obviously of great importance, both theoretically and for applications, not only to find a solution but also to detect its shape and other qualitative properties. In particular, for domains with corners, it is very interesting to find solutions which are localized at the corners as, often, one can guess from applications or from energy considerations.

Here, by computer-assistance, we prove the existence of such a solution which is close to an approximate numerically computed solution with the desired shape. The method and the precise result will be stated in the next section.

Moreover, solutions in unbounded domains can be used to find solutions in bounded domains by perturbative methods. Roughly speaking in some nonlinear parameter dependent elliptic problems in bounded domains which, as the parameter goes to a limit value, "tend" to a similar problem in an unbounded domain, one can use the solution in the unbounded domain to construct solutions of the original problem. This is usually done by "gluing" together several 
"copies" of suitable truncations of the solution in the unbounded domain. This technique usually requires two main properties of the solution in the unbounded domain:

i) it must be nondegenerate,

ii) it should have a suitably fast decay at infinity.

Some typical cases, widely analyzed in the literature, are so-called singularly-perturbed problems or problems with critical nonlinearities. In a similar way a solution of (1) can be used to find solutions in expanding bounded tubular domains.

Let us be more precise. Let $M$ be a compact $k$-dimensional piecewise smooth submanifold of $\mathbb{R}^{N}$ without boundary, $1 \leq k \leq N-1, N \geq 2$. For $R>0$ define the expanded manifold $M_{R}=\{R x, x \in M\}$ and denote by $\Omega_{R}$ its open tubular neighborhood of radius 1 . Then consider the problem

$$
\left\{\begin{aligned}
-\Delta u & =f(u) & & \text { in } \Omega_{R} \\
u & =0 & & \text { on } \partial \Omega_{R}
\end{aligned}\right.
$$

for some nonlinear $C^{1}$-function $f$. The equation (3) appears e.g. in nonlinear optics and models standing waves in optical waveguides. The most interesting variant for applications which exploits the nonlinear properties of the material is the self-focusing case where $f(u) / u \rightarrow \infty$ as $|u| \rightarrow \infty$. A typical example is given by $f(u)=u^{3}$, modelling Kerr's effect. For more information on the physical background see for example [22].

When $k=1$ then $\Omega_{R}$ is a tubular guide, i.e. an optical fiber and the flat case, $N=2$, is an interesting one. When $k \geq 2$ and $M$ is regular and symmetric, in particular $\Omega_{R}$ is an expanding annuli, some existence results have been found using variational methods ([10], [4], [17], [23]).

The first paper which deals with the nonsymmetric case, but always assuming $M$ to be regular, is [1], where solutions are found in the form

$$
u_{R}=\sum_{i=1}^{m} U_{X_{R, i}, R}+o(1)
$$

in $H^{1}\left(\mathbb{R}^{N}\right)$ as $R \rightarrow \infty$, where $U_{X_{R, i}, R}$ are solutions of the "local" limit problem in an open unbounded cylinder centered at suitable points $X_{R, i}, i=1, \ldots, m$ of the domain $\Omega_{R}$. Solutions of the form (44) are usually called "multibump" solutions. This result was improved in [1] for positive solutions and extended then to sign-changing solutions in [2] in the case $k=1$, but always starting from regular manifolds.

If the original 1-dimensional manifold has some "corners" one would expect the existence of similar "multibump" solutions, but with (some) bumps localized at the corners. In order to find such a type of solutions the main thing is to have a solution of a limit problem in an unbounded domain with a corner which is precisely localized at a corner. More precisely, if the 1-dimensional manifold $M$ is a piecewise regular manifold in the plane given by the union of a finite number of smooth curves which intersect orthogonally one could use the solution we find in the $L$-shaped domain $\Omega$ to construct a multibump solution of type (44) with the bumps (positive or negative) at the corners.

It is important at this point to stress what we have already mentioned in (2), i.e. that in order to use the solution $u$ in the unbounded $L$-shaped domain as building block for solutions in 
expanding bounded domains with corners, we need the two main properties i) and ii) (see (2)). In other words the solution $u$ must be nondegenerate and decay exponentially at infinity.

In this paper we also prove both properties of the solution found by a computer-assisted proof and we compute its Morse index (which is one). We believe that all these results are interesting in themselves.

Finally, let us explain briefly the numerical and analytical techniques that we use for our computer-assisted proof. We start with an approximate solution $\omega \in H_{0}^{1}(\Omega)$ to problem (1) which we compute by a Newton iteration combined with a finite element method; in order to overcome the singularity problems at the re-entrant corner $(0,0)$ of $\Omega$, we also involve the associated singularity function into the approximation process. In this first approximative step, there is no need for any mathematical rigor.

Now we put up a boundary value problem for the error $v=u-w$, which we re-write as a fixed-point equation involving the residual $-\Delta \omega-|\omega|^{3}$ of the approximate solution, and the inverse of the linearization $L_{\omega}=-\Delta-3|\omega| \omega$. Now Banach's Fixed-Point Theorem gives the existence of a solution to problem (1) in some "close" $H_{0}^{1}(\Omega)$-neighborhood of $\omega$, provided that the residual is sufficiently small (measured in $H^{-1}(\Omega)$ ) and $\left\|L_{\omega}^{-1}\right\|_{H^{-1}(\Omega) \rightarrow H_{0}^{1}(\Omega)}$ is "moderate"; the precise conditions are formulated in Theorem 1.

For computing a rigorous bound to the residual we use an additional $H(\operatorname{div}, \Omega$ )-approximation to $\nabla \omega$ (see Section 4) and rigorous bounds to some integrals. A norm bound for $L_{\omega}^{-1}$ is obtained via spectral bounds for $L_{\omega}$ : The essential spectrum can be bounded by simple Rayleigh quotient estimates, and isolated eigenvalues below the essential spectrum are enclosed by variational methods and additional computer-assisted means, supplemented by a homotopy method for obtaining some necessary spectral a priori information.

By related computer-assisted techniques we have been able to prove existence, multiplicity, and also uniqueness statements for various kinds of boundary and eigenvalue problems; see e.g. [6, 9, 14, 18, 19, 20].

The paper is organized as follows. In Section 2 we formulate the basic theorem (Theorem 1) for our computer-assisted proof. Section 3 contains a description of the numerical methods used to obtain an approximate solution $\omega$, and Section 4 the essential estimates for obtaining a residual bound. Section 5 is devoted to the spectral estimates for $L_{\omega}$ needed to bound $\left\|L_{\omega}^{-1}\right\|_{H^{-1}(\Omega) \rightarrow H_{0}^{1}(\Omega)}$. Section 6 contains some more numerical details and our computational results proving the existence of a solution to problem (11) together with a close $H_{0}^{1}(\Omega)$-error bound. Based on the spectral information obtained as a "side product" of our computerassisted proof, we prove nondegeneracy of the solution in Section 7. Finally, Section 8 contains a proof of exponential decay of the solution.

\section{Existence and Enclosure Theorem}

Let $\Omega=((-1, \infty) \times(0,1)) \cup((-1,0) \times(-\infty, 1))$, i.e. $\Omega$ is an unbounded L-shaped domain. Let $H_{0}^{1}(\Omega)$ be endowed with the inner product $\langle u, v\rangle_{H_{0}^{1}}:=\langle\nabla u, \nabla v\rangle_{L^{2}}+\langle u, v\rangle_{L^{2}}$, and let $H^{-1}(\Omega)$ denote the dual space of $H_{0}^{1}(\Omega)$, equipped with the usual operator sup-norm. We 
consider the problem

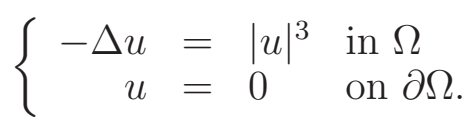

Our goal is to prove existence of a non-trivial solution by computer-assistance.

We assume that $\omega \in H_{0}^{1}(\Omega)$ is an approximate solution to (5) (computed numerically) and that constants $\delta$ and $K$ are known such that

(a) $\delta$ bounds the defect (residual) of the approximate solution in the $H^{-1}$-norm, i.e.

$$
\left\|-\Delta \omega-|\omega|^{3}\right\|_{H^{-1}} \leq \delta
$$

(b) $K$ bounds the inverse of the linearization at $\omega$, i.e.

$$
\|v\|_{H_{0}^{1}} \leq K\left\|L_{\omega}[v]\right\|_{H^{-1}} \quad \text { for all } v \in H_{0}^{1}(\Omega)
$$

where $L_{\omega}: H_{0}^{1}(\Omega) \rightarrow H^{-1}(\Omega), L_{\omega}[v]=-\Delta v-3|\omega| \omega v$ denotes the linearized operator.

Note that condition (17) implies that $L_{\omega}$ is one-to-one. We will also need that $L_{\omega}$ is onto. For proving this we use the canonical isometric isomorphism $\Phi: H_{0}^{1}(\Omega) \rightarrow H^{-1}(\Omega)$ given by

$$
(\Phi[u])(v):=\langle u, v\rangle_{H_{0}^{1}} \quad\left(u, v \in H_{0}^{1}(\Omega)\right)
$$

and show that

(i) $\left(\Phi^{-1} L_{\omega}\right)\left(H_{0}^{1}(\Omega)\right)$ is dense in $H_{0}^{1}(\Omega)$ (implying that $L_{\omega}\left(H_{0}^{1}(\Omega)\right) \subset H^{-1}(\Omega)$ is dense),

(ii) $L_{\omega}\left(H_{0}^{1}(\Omega)\right) \subset H^{-1}(\Omega)$ is closed.

For proving (i) we first note that $\Phi^{-1} L_{\omega}: H_{0}^{1}(\Omega) \rightarrow H_{0}^{1}(\Omega)$ is symmetric w.r.t. $\langle\cdot, \cdot\rangle_{H_{0}^{1}}$ :

$$
\left\langle\Phi^{-1} L_{\omega}[u], v\right\rangle_{H_{0}^{1}} \stackrel{(\underline{8})}{=}\left(L_{\omega}[u]\right)[v]=\int_{\Omega} \nabla u \cdot \nabla v-3|\omega| \omega u v d x
$$

Let now $u \in H_{0}^{1}(\Omega)$ be an element of the orthogonal complement of $\left(\Phi^{-1} L_{\omega}\right)\left(H_{0}^{1}(\Omega)\right)$, i.e. we have

$$
0=\left\langle u, \Phi^{-1} L_{\omega}[v]\right\rangle_{H_{0}^{1}} \stackrel{\text { symmetry }}{=}\left\langle\Phi^{-1} L_{\omega}[u], v\right\rangle_{H_{0}^{1}} \quad \text { for all } v \in H_{0}^{1}(\Omega) .
$$

Therefore $\Phi^{-1} L_{\omega}[u]=0$, which implies $L_{\omega}[u]=0$ and, since $L_{\omega}$ is one-to-one, $u=0$. Thus (i) follows.

To prove (ii), let $\left(L_{\omega}\left[u_{n}\right]\right)_{n \in \mathbb{N}}$ be a sequence in $L_{\omega}\left(H_{0}^{1}(\Omega)\right)$ converging to some $r \in H^{-1}(\Omega)$. Condition (7) shows that $\left(u_{n}\right)_{n \in \mathbb{N}}$ is a Cauchy sequence in $H_{0}^{1}(\Omega)$ and thus converges to some $u \in H_{0}^{1}(\Omega)$. Since $L_{\omega}$ is bounded we obtain $L_{\omega}\left[u_{n}\right] \rightarrow L_{\omega}[u] \quad(n \rightarrow \infty)$, which gives $r=L_{\omega}[u] \in$ $L_{\omega}\left(H_{0}^{1}(\Omega)\right)$ and therefore the closedness of $L_{\omega}\left(H_{0}^{1}(\Omega)\right)$ in $H^{-1}(\Omega)$. 
Theorem 1. Let $\omega \in H_{0}^{1}(\Omega)$ be an approximate solution to (5), and $\delta$ and $K$ constants such that (6) and (7) are satisfied. Let moreover $C_{4}>0$ be an embedding constant for the embedding $H_{0}^{1}(\Omega) \hookrightarrow L^{4}(\Omega)$, and $\gamma:=3 C_{4}^{3}$.

Finally suppose that there exists some $\alpha>0$ such that

$$
\delta \leq \frac{\alpha}{K}-\gamma \alpha^{2}\left(\|\omega\|_{L^{4}}+\frac{1}{3} C_{4} \alpha\right)
$$

and

$$
2 K \gamma \alpha\left(\|\omega\|_{L^{4}}+\frac{1}{2} C_{4} \alpha\right)<1 .
$$

Then there exists a solution $u \in H_{0}^{1}(\Omega)$ to problem (5) such that

$$
\|u-\omega\|_{H_{0}^{1}} \leq \alpha .
$$

In particular, $u$ is non-trivial if $\alpha<\|\omega\|_{H_{0}^{1}}$.

Remark 1. (a) Let $\psi(\alpha)$ denote the right-hand-side of (10), which obviously attains a positive maximum on $[0, \infty)$. Thus the existence of some $\alpha>0$ satisfying (10) is equivalent to

$$
\delta \leq \max _{\alpha \in[0, \infty)} \psi(\alpha),
$$

which due to (6) will be satisfied if the approximate solution $\omega$ is computed with sufficiently high accuracy. Furthermore, a small defect bound $\delta$ will imply a small error bound $\alpha$ if $K$ is not too large.

(b) As shown in [18], (10) implies (11) if $\delta$ satisfies (13) with a strict inequality and $\alpha$ is chosen appropriately.

For the proof of Theorem 1 see [18. It is based on Banach's Fixed Point Theorem and uses the following Lemma (see [18, Lemma 3.1 and 3.2]), which in addition will be useful in a later section of this paper.

Lemma 1. Let $p_{1}, p_{2}, p_{3}, p_{4} \in[2, \infty)$ such that $\frac{1}{p_{1}}+\frac{1}{p_{2}}+\frac{1}{p_{3}}+\frac{1}{p_{4}}=1$ and $C_{p_{i}}>0$ an embedding constant for the embedding $H_{0}^{1}(\Omega) \hookrightarrow L^{p_{i}}(\Omega), i=1, \ldots, 4$. Then (i) and (ii) hold true:

(i) For all $u, \tilde{u}, v \in H_{0}^{1}(\Omega)$ :

$$
\|[|u| u-|\tilde{u}| \tilde{u}] v\|_{H^{-1}} \leq C_{p_{3}} C_{p_{4}}\left(\|u\|_{L^{p_{1}}}+\|\tilde{u}\|_{L^{p_{1}}}\right)\|u-\tilde{u}\|_{L^{p_{2}}}\|v\|_{H_{0}^{1}} .
$$

(ii) Let $u, \tilde{u} \in H_{0}^{1}(\Omega)$, and let $L_{u}$ and $L_{\tilde{u}}$ denote the linearizations at $u$ and $\tilde{u}$, respectively. Suppose that for some $K>0$

$$
\|v\|_{H_{0}^{1}} \leq K\left\|L_{\tilde{u}}[v]\right\|_{H^{-1}} \quad \text { for all } v \in H_{0}^{1}(\Omega)
$$

and

$$
\kappa:=3 C_{p_{3}} C_{p_{4}} K\left(\|u\|_{L^{p_{1}}}+\|\tilde{u}\|_{L^{p_{1}}}\right)\|u-\tilde{u}\|_{L^{p_{2}}}<1
$$

Then,

$$
\|v\|_{H_{0}^{1}} \leq \frac{K}{1-\kappa}\left\|L_{u}[v]\right\|_{H^{-1}} \quad \text { for all } v \in H_{0}^{1}(\Omega) .
$$

Remark 2. Note that, in (14), $\|u-\tilde{u}\|_{L^{p_{2}}}$ can be replaced by $C_{p_{2}}\|u-\tilde{u}\|_{H_{0}^{1}}$, which for the particular choice $p_{1}=p_{2}=p_{3}=p_{4}=4$ amounts to the condition

$$
\kappa:=\gamma K\left(\|u\|_{L^{4}}+\|\tilde{u}\|_{L^{4}}\right)\|u-\tilde{u}\|_{H_{0}^{1}}<1 .
$$




\section{Computation of an approximate solution}

Let $T>1$ and define $\Omega_{0}:=\Omega \cap(-T, T)^{2}$. Then $\Omega_{0}$ is bounded and contains the corner-part of $\Omega$. Let $\omega_{0} \in H_{0}^{1}\left(\Omega_{0}\right)$ be an approximate solution of

$$
\left\{\begin{aligned}
-\Delta u & =|u|^{3} & & \text { in } \Omega_{0} \\
u & =0 & & \text { on } \partial \Omega_{0} .
\end{aligned}\right.
$$

Then

$$
\omega=\left\{\begin{array}{ccc}
\omega_{0} & \text { in } & \Omega_{0} \\
0 & \text { in } & \Omega \backslash \Omega_{0}
\end{array}\right.
$$

is in $H_{0}^{1}(\Omega)$ and turns out to be a good approximate solution of (5) if $T$ is chosen large enough and $\omega_{0}$ is sufficiently accurate. Indeed our numerical results show that $T=3$ is sufficient.

An approximate solution of (15) can be computed using finite elements and a Newton iteration. As an initial guess, an appropriate multiple of the first eigenfunction of $-\Delta$ in $(-1,0) \times(0,1)$ with homogeneous Dirichlet boundary conditions and extended by zero to $\Omega_{0} \backslash((-1,0) \times(0,1))$ can be used. However, due to the re-entrant corner at 0 , approximations obtained with finite elements alone do not yield a sufficiently small defect. So in addition we use a corner singular function, which allows us to write the exact solution of (15) as a sum of a singular part and a regular part in $H^{2}\left(\Omega_{0}\right)$ (see [12] and [13]): We introduce polar coordinates $(r, \varphi)$, where $r=|x|$ and $\varphi$ ranges between 0 and $\theta:=\frac{3 \pi}{2}$, with $\varphi=0$ for $x>0, y=0$ and $\varphi=\theta$ for $x=0, y<0$. On $\bar{\Omega}_{0}$ we define

$$
\gamma(r, \varphi):=r^{\frac{2}{3}} \sin \left(\frac{2}{3} \varphi\right)
$$

Obviously, $\gamma(r, 0)=\gamma(r, \theta)=0$ and one can easily check that $\Delta \gamma=0$ in $\Omega_{0}$. We choose some fixed function $\lambda \in H^{2}\left(\Omega_{0}\right) \cap C^{1}\left(\bar{\Omega}_{0}\right)$ which vanishes on the part of $\partial \Omega_{0}$ where $\gamma$ does not vanish and satisfies $\lambda(0)=1, \nabla \lambda(0)=0$. With $w:=\lambda \gamma \in H_{0}^{1}\left(\Omega_{0}\right)$, a solution $u \in H_{0}^{1}\left(\Omega_{0}\right)$ to (15) can then be written as

$$
u=a w+v,
$$

where $v \in H^{2}\left(\Omega_{0}\right) \cap H_{0}^{1}\left(\Omega_{0}\right)$ is the regular part and $a \in \mathbb{R}$ is the so-called stress-intensity-factor. Using the dual singular function $\Gamma(r, \varphi):=r^{-\frac{2}{3}} \sin \left(\frac{2}{3} \varphi\right)$ we can represent $a$ by means of the solution $u$, i.e. we have

$$
a=\frac{1}{\pi}\left(\int_{\Omega_{0}}(\Lambda \Gamma)|u|^{3}+\Delta(\Lambda \Gamma) u d x\right),
$$

where $\Lambda \in H^{2}\left(\Omega_{0}\right) \cap C^{1}\left(\bar{\Omega}_{0}\right)$ is a cutoff function with similar properties as $\lambda$ and such that $\Delta(\Lambda \Gamma) \in L^{2}\left(\Omega_{0}\right)$. A suitable choice for $\lambda$ and $\Lambda$ is e.g. given by $\lambda(x, y)=\Lambda(x, y)=(1-$ $\left.x^{2}\right)^{2}\left(1-y^{2}\right)^{2} \chi_{(-1,1)^{2}}(x, y)$ for $(x, y) \in \bar{\Omega}_{0}$.

Clearly, a computation of the exact stress-intensity-factor by (19) is impossible since the exact solution $u$ is unknown. For our purpose - the improvement of the approximate solution - it is however sufficient to know only an approximation of $a$. So let a finite element function $\tilde{\omega}_{0}$ (computed without separate singular part) be an approximate solution of (15). Inserting $\tilde{\omega}_{0}$ into (19) yields an approximate stress-intensity-factor

$$
\tilde{a}:=\frac{1}{\pi} \int_{\Omega_{0}}(\Lambda \Gamma)\left|\tilde{\omega}_{0}\right|^{3}+\Delta(\Lambda \Gamma) \tilde{\omega}_{0} d x .
$$


The regular part $v=u-a w \in H^{2}\left(\Omega_{0}\right) \cap H_{0}^{1}\left(\Omega_{0}\right)$ of a solution $u$ to (15) satisfies

$$
-\Delta v=|a w+v|^{3}+a \Delta w
$$

and thus an approximate regular part $\tilde{v}$ (in the finite element space) can be computed using a Newton iteration with initial guess $v_{0}=\tilde{\omega}_{0}-\tilde{a} I(w)$, where $I$ denotes the interpolation operator for the finite element space. Our new approximate solution to (15) is then given by

$$
\omega_{0}=\tilde{a} w+\tilde{v}
$$

which yields an approximate solution $\omega$ to (15) by (16).

\section{Computation of a residual bound}

Let $\tilde{\rho} \in H(\operatorname{div}, \Omega)=\left\{v \in\left(L^{2}(\Omega)\right)^{2}: \operatorname{div} v \in L^{2}(\Omega)\right\}$ be an approximation of $\nabla \omega$, such that $\tilde{\rho}$ is also an approximate solution of $\operatorname{div} \rho=-|\omega|^{3}$. We comment on the computation of $\tilde{\rho}$ in subsection 6.1. Then we can estimate:

$$
\begin{aligned}
\left\|-\Delta \omega-|\omega|^{3}\right\|_{H^{-1}} & \leq\|-\operatorname{div}(\nabla \omega-\tilde{\rho})\|_{H^{-1}}+\left\|-\operatorname{div} \tilde{\rho}-|\omega|^{3}\right\|_{H^{-1}} \\
& \leq\|\nabla \omega-\tilde{\rho}\|_{L^{2}}+C_{2}\left\|-\operatorname{div} \tilde{\rho}-|\omega|^{3}\right\|_{L^{2}},
\end{aligned}
$$

with $C_{2}$ denoting an embedding constant for the embedding $H_{0}^{1}(\Omega) \hookrightarrow L^{2}(\Omega)$ and hence also for $L^{2}(\Omega) \hookrightarrow H^{-1}(\Omega)$. Now we are left to compute upper bounds just for integrals, which due to the splitting of the approximate solution into singular and regular part is however still technically a bit challenging. We will comment on this in section 6.1.

Remark 3. If $-\Delta \omega-|\omega|^{3} \in L^{2}(\Omega)$ (which requires higher smoothness of the approximate solution), one can also use the embedding $L^{2}(\Omega) \hookrightarrow H^{-1}(\Omega)$ directly to compute an upper bound for the residuum:

$$
\left\|-\Delta \omega-|\omega|^{3}\right\|_{H^{-1}} \leq C_{2}\left\|-\Delta \omega-|\omega|^{3}\right\|_{L^{2}}
$$

Again, it remains to compute bounds for an integral.

\section{Computation of $\mathrm{K}$}

We use the isometric isomorphism $\Phi: H_{0}^{1}(\Omega) \rightarrow H^{-1}(\Omega)$, defined in (8), to obtain

$$
\left\|L_{\omega}[u]\right\|_{H^{-1}}=\left\|\left(\Phi^{-1} L_{\omega}\right)[u]\right\|_{H_{0}^{1}} \quad\left(u \in H_{0}^{1}(\Omega)\right)
$$

and therefore

$$
\|v\|_{H_{0}^{1}} \leq K\left\|L_{\omega}[v]\right\|_{H^{-1}} \quad\left(v \in H_{0}^{1}(\Omega)\right) \Longleftrightarrow\|v\|_{H_{0}^{1}} \leq K\left\|\left(\Phi^{-1} L_{\omega}\right)[v]\right\|_{H_{0}^{1}} \quad\left(v \in H_{0}^{1}(\Omega)\right) .
$$

Moreover, (9) already showed that $\Phi^{-1} L_{\omega}: H_{0}^{1}(\Omega) \rightarrow H_{0}^{1}(\Omega)$ is symmetric and due to its definition on the whole space $H_{0}^{1}(\Omega)$ therefore self-adjoint. Thus (22) holds for any

$$
K \geq \frac{1}{\min \left\{|\nu|: \quad \nu \text { is in the spectrum of } \Phi^{-1} L_{\omega}\right\}},
$$


provided the minimum is positive. We are therefore left to compute bounds for the essential spectrum of the operator $\Phi^{-1} L_{\omega}$ as well as bounds for the eigenvalues of finite multiplicity which are closest to 0 . We first draw our attention to the essential spectrum.

Consider the operator $L_{0}: H_{0}^{1}(\Omega) \rightarrow H^{-1}(\Omega), v \mapsto-\Delta v+\left(\frac{\pi^{2}}{\pi^{2}+1} \chi_{\Omega_{1}}\right) v$, with $\chi_{\Omega_{1}}$ denoting the characteristic function on $\Omega_{1}:=(-1,0) \times(0,1)$. Since both $\omega$ and $\chi_{\Omega_{1}}$ have compact support and are bounded, $\Phi^{-1} L_{\omega}-\Phi^{-1} L_{0}: H_{0}^{1}(\Omega) \rightarrow H_{0}^{1}(\Omega)$ is compact, and hence $\sigma_{\text {ess }}\left(\Phi^{-1} L_{\omega}\right)=$ $\sigma_{\text {ess }}\left(\Phi^{-1} L_{0}\right)$ due to a well-known perturbation result [15]. To bound $\sigma_{\text {ess }}\left(\Phi^{-1} L_{0}\right)$ we consider Rayleigh quotients: $\Omega \backslash \Omega_{1}$ is the union of two disjoint semi-infinite strips, on each of which the Rayleigh quotient $\frac{\|\nabla u\|_{L^{2}}^{2}}{\|u\|_{L^{2}}^{2}}$ is bounded from below by $\pi^{2}$. Hence, for each $u \in H_{0}^{1}(\Omega)$,

$$
\int_{\Omega \backslash \Omega_{1}}|\nabla u|^{2} d x \geq \frac{\pi^{2}}{\pi^{2}+1} \int_{\Omega \backslash \Omega_{1}}\left[|\nabla u|^{2}+u^{2}\right] d x .
$$

Furthermore, trivially

$$
\int_{\Omega_{1}}\left[|\nabla u|^{2}+\frac{\pi^{2}}{\pi^{2}+1} u^{2}\right] d x \geq \frac{\pi^{2}}{\pi^{2}+1} \int_{\Omega_{1}}\left[|\nabla u|^{2}+u^{2}\right] d x
$$

holds. Adding (24) and (25) gives, for each $u \in H_{0}^{1}(\Omega)$,

$$
\int_{\Omega}\left[|\nabla u|^{2}+\left(\frac{\pi^{2}}{\pi^{2}+1} \chi_{\Omega_{1}}\right) u^{2}\right] d x \geq \frac{\pi^{2}}{\pi^{2}+1}\langle u, u\rangle_{H_{0}^{1}},
$$

and the left-hand side equals $\left\langle\Phi^{-1} L_{0} u, u\right\rangle_{H_{0}^{1}}$. So the Rayleigh quotient, and hence the spectrum, and in particular the essential spectrum of $\Phi^{-1} L_{0}$ is bounded from below by $\frac{\pi^{2}}{\pi^{2}+1}$. Hence also $\sigma_{\mathrm{ess}}\left(\Phi^{-1} L_{\omega}\right) \subset\left[\frac{\pi^{2}}{\pi^{2}+1}, \infty\right)$.

For analyzing eigenvalues of $\Phi^{-1} L_{\omega}$ we note that, for $(\nu, u) \in \mathbb{R} \times H_{0}^{1}(\Omega), \nu \neq 1$,

$$
\begin{aligned}
\left(\Phi^{-1} L_{\omega}\right)[u]=\nu u & \Longleftrightarrow L_{\omega}[u]=\nu \Phi[u] \\
& \Longleftrightarrow-\Delta u-3|\omega| \omega u=\nu(-\Delta u+u) \\
& \Longleftrightarrow(1-\nu)(-\Delta u+u)=(1+3|\omega| \omega) u \\
& \Longleftrightarrow(-\Delta u+u)=\underbrace{\frac{1}{1-\nu}(1+3|\omega| \omega) u,}_{=: \kappa} \\
& \Longleftrightarrow \underbrace{\int_{\Omega}[\nabla u \cdot \nabla \varphi+u \varphi] d x}_{=\langle u, \varphi\rangle_{H_{0}^{1}}}=\kappa \underbrace{\int_{\Omega}(1+3|\omega| \omega) u \varphi d x}_{=: N(u, \varphi)} \quad \text { for all } \varphi \in H_{0}^{1}(\Omega),
\end{aligned}
$$

which gives a new eigenvalue problem avoiding $\Phi^{-1}$, with spectral parameter $\kappa . N$ is a symmetric bilinear form on $H_{0}^{1}(\Omega)$ and due to the positivity of $\omega$, which can be proved by interval evaluations, also positive definite. Therefore, $1-\nu>0$ for all possible eigenvalues $\nu$ and 
we are now left to compute upper and lower bounds for eigenvalues $\kappa$ of (26) neighbouring 1. Defining the essential spectrum of (26) in the usual way to be the one of its associated self-adjoint operator $R=\left(I_{H_{0}^{1}}-\Phi^{-1} L_{\omega}\right)^{-1}$, we see that it is bounded from below by $\left(1-\min \sigma_{\text {ess }}\left(\Phi^{-1} L_{\omega}\right)\right)^{-1} \geq \pi^{2}+1$.

The following theorem is well known and provides an easy and efficient way for computing upper bounds to eigenvalues below the essential spectrum, and hence (here) in particular to eigenvalues below $\pi^{2}+1$ :

Theorem 2 (Rayleigh-Ritz). Let $v_{1}, \ldots, v_{n} \in H_{0}^{1}(\Omega)$ be linearly independent and define the matrices

$$
A_{0}:=\left(\left\langle v_{i}, v_{j}\right\rangle_{H_{0}^{1}}\right)_{i, j=1, \ldots, n}, \quad A_{1}:=\left(N\left(v_{i}, v_{j}\right)\right)_{i, j=1, \ldots, n} .
$$

Denote by $\Lambda_{1} \leq \ldots \leq \Lambda_{n}$ the eigenvalues of $A_{0} x=\Lambda A_{1} x$ and suppose that $\Lambda_{n}<\pi^{2}+1$. Then there are at least $n$ eigenvalues of (26) below $\pi^{2}+1$, and the $n$ smallest of these, ordered by magnitude, satisfy

$$
\kappa_{i} \leq \Lambda_{i}, \quad i=1, \ldots, n .
$$

Note that good upper bounds will be obtained by Theorem 2 if $v_{1}, \ldots, v_{n} \in H_{0}^{1}(\Omega)$ are chosen as approximate eigenfunctions associated with the $n$ smallest eigenvalues of (26) $)$. The remaining task for applying Theorem 2 is the enclosure of matrix eigenvalues, which can be achieved using interval arithmetic and [14, Lemma 4] or using interval packages like INTLAB [21].

For the computation of lower eigenvalue bounds, which is more problematic than obtaining upper bounds, we use the following method of Lehmann and Goerisch (see [5]).

Theorem 3. Let $v_{1}, \ldots, v_{n} \in H_{0}^{1}(\Omega)$ and $A_{0}, A_{1}$ as before. Let $X$ be some vector space, $b$ some symmetric, positive definite bilinear form on $X$, and $T: H_{0}^{1}(\Omega) \rightarrow X$ some linear operator satisfying $b(T \psi, T \varphi)=\langle\psi, \varphi\rangle_{H_{0}^{1}}$ for all $\psi, \varphi \in H_{0}^{1}(\Omega)$.

Let $w_{1}, \ldots, w_{n} \in X$ satisfy

$$
b\left(T \varphi, w_{i}\right)=N\left(\varphi, v_{i}\right) \quad \text { for all } \varphi \in H_{0}^{1}(\Omega), i=1, \ldots, n
$$

and define $A_{2}:=\left(b\left(w_{i}, w_{j}\right)\right)_{i, j=1 \ldots, n}$. Moreover, let $\rho \in \mathbb{R}$ such that

$$
\Lambda_{n}<\rho \leq \pi^{2}+1
$$

and in addition

$$
\rho \leq \kappa_{n+1},
$$

if an $(n+1)$-st eigenvalue $\kappa_{n+1}<\pi^{2}+1$ exists.

Then, with $\mu_{1} \leq \ldots \leq \mu_{n}<0$ denoting the eigenvalues of

$$
\left(A_{0}-\rho A_{1}\right) x=\mu\left(A_{0}-2 \rho A_{1}+\rho^{2} A_{2}\right) x,
$$

we have

$$
\kappa_{m} \geq \rho-\frac{\rho}{1-\mu_{n+1-m}}, \quad m=1, \ldots, n .
$$


Remark 4. (i) (28) and Theorem 2 imply in particular that at least $n$ eigenvalues $\kappa_{1} \leq$ $\ldots \leq \kappa_{n}<\pi^{2}+1$ exist.

(ii) Again by (28) and Theorem 2, the matrix $A_{0}-\rho A_{1}$ is negative definite. Moreover, (27) and (28) show after some calculations that $A_{0}-2 \rho A_{1}+\rho^{2} A_{2}$ is positive definite, hence the matrix eigenvalue problem (30) has indeed only negative eigenvalues.

(iii) We will see later that usually (27) does not determine $w_{1}, \ldots, w_{n}$ uniquely. A closer look at the proof of Theorem 3 makes clear that good bounds will be obtained if $w_{i} \approx \frac{1}{\Lambda_{i}} T v_{i}$, when $\left(\Lambda_{i}, v_{i}\right)$ is an approximate eigenpair to problem (26).

(iv) Condition (29) requires an a-priori lower bound for the $(n+1)$-st eigenvalue (if it exists) in order to compute lower bounds for the $n$ smallest eigenvalues. However, a rough lower bound $\rho$ will be sufficient for this purpose and can be obtained using a homotopy method (see subsection 5.1).

We will now explain how to choose $X, b, T$ and $w_{1}, \ldots, w_{n} \in X$ satisfying the assumptions of Theorem 3; Let

$$
X=\left(L^{2}(\Omega)\right)^{2} \times L^{2}(\Omega), b\left(\left(\begin{array}{c}
w_{1} \\
w_{2}
\end{array}\right),\left(\begin{array}{c}
\tilde{w}_{1} \\
\tilde{w}_{2}
\end{array}\right)\right):=\left\langle w_{1}, \tilde{w}_{1}\right\rangle_{L^{2}}+\left\langle w_{2}, \tilde{w}_{2}\right\rangle_{L^{2}}, T u:=\left(\begin{array}{c}
\nabla u \\
u
\end{array}\right) .
$$

Obviously, $b(T \psi, T \varphi)=\langle\psi, \varphi\rangle_{H_{0}^{1}}$ for all $\psi, \varphi \in H_{0}^{1}(\Omega)$. We consider condition (27):

$$
\begin{aligned}
& b\left(T \varphi, w_{i}\right)=N\left(\varphi, v_{i}\right) \quad \text { for all } \varphi \in H_{0}^{1}(\Omega) \\
\Longleftrightarrow & \left\langle\nabla \varphi, w_{i, 1}\right\rangle_{L^{2}}+\left\langle\varphi, w_{i, 2}\right\rangle_{L^{2}}=\left\langle\varphi,(1+3|\omega| \omega) v_{i}\right\rangle_{L^{2}} \quad \text { for all } \varphi \in H_{0}^{1}(\Omega) \\
\Longleftrightarrow & w_{i, 1} \in H(\operatorname{div}, \Omega), \quad-\operatorname{div}\left(w_{i, 1}\right)+w_{i, 2}=(1+3|\omega| \omega) v_{i} \\
\Longleftrightarrow & w_{i, 1} \in H(\operatorname{div}, \Omega), \quad w_{i, 2}=\operatorname{div}\left(w_{i, 1}\right)+(1+3|\omega| \omega) v_{i} .
\end{aligned}
$$

This shows that $w_{i, 1} \in H(\operatorname{div}, \Omega)$ can be chosen arbitrarily whereas $w_{i, 2}$ has to be chosen according to (31). Recalling Remark 4 (iii), one should have $w_{i} \approx \frac{1}{\Lambda_{i}} T v_{i}$ with an approximate eigenpair $\left(\Lambda_{i}, v_{i}\right)$ to obtain good bounds. Since $w_{i, 2}$ is already fixed, it remains to require

$$
w_{i, 1} \approx \frac{1}{\Lambda_{i}} \nabla v_{i}
$$

A suitable choice of $w_{i, 1}$ is therefore given by an approximate minimizer in $H(\operatorname{div}, \Omega)$ of

$$
\left\|\frac{1}{\Lambda_{i}} \nabla v_{i}-w\right\|_{L^{2}}^{2}+\left\|-\operatorname{div} w+\left(\frac{1}{\Lambda_{i}}-(1+3|\omega| \omega)\right) v_{i}\right\|_{L^{2}}^{2} .
$$

\subsection{Homotopy method}

Our aim is now to find some $\rho \in \mathbb{R}$ such that $\Lambda_{n}<\rho \leq \kappa_{n+1}<\pi^{2}+1$ if $\kappa_{n+1}$ exists, or $\Lambda_{n}<\rho \leq \pi^{2}+1$ otherwise. The crucial idea is to find a base problem, for which we have knowledge about the eigenvalues, and connect it with the original problem via a family of eigenvalue problems such that, indexwise, the eigenvalues increase along the homotopy. In our case it is necessary to combine two separate homotopies: One is needed to find lower bounds 
for eigenvalues of the eigenvalue problem $-\Delta u+u=\kappa(1+\bar{c}) u$ in $\Omega\left(u \in H_{0}^{1}(\Omega)\right)$, where $\bar{c}$ is a suitable piecewise constant function on $\Omega$. A second homotopy then connects this eigenvalue problem to the original one. For the first homotopy we use a domain decomposition method, which goes back to an idea of E.B. Davies and is explained in detail in [6].

To construct a suitable base problem, choose $0=\xi_{0}<\xi_{1}<\ldots<\xi_{k}$ and a function $\bar{c} \geq 3|\omega| \omega$ which is constant on each of the rectangles

$$
\begin{aligned}
& (-1,0) \times(0,1), \quad\left(\xi_{i}, \xi_{i+1}\right) \times(0,1) \quad(i=0, \ldots, k-1), \quad\left(\xi_{k}, \infty\right) \times(0,1) \\
& (-1,0) \times\left(-\xi_{i+1},-\xi_{i}\right) \quad(i=0, \ldots, k-1), \quad(-1,0) \times\left(-\infty,-\xi_{k}\right) .
\end{aligned}
$$

Note that since $\omega$ has compact support, $\bar{c}=0$ on $\left(\xi_{k}, \infty\right) \times(0,1)$ and on $(-1,0) \times\left(-\infty,-\xi_{k}\right)$ can be chosen if $\xi_{k}$ is large enough (which we will assume in the following). Define now $\Omega_{1}:=(-1,0) \times(0,1), \Omega_{2}:=(0, \infty) \times(0,1), \Omega_{3}:=(-1,0) \times(-\infty, 0)$ and consider for $j=1,2,3$ the eigenvalue problems

$$
\left\{\begin{aligned}
-\Delta u+u & =\kappa(1+\bar{c}) u & & \text { in } \Omega_{j} \\
\frac{\partial u}{\partial \nu} & =0 & & \text { on the interfaces } \Gamma_{1}:=\{0\} \times(0,1), \Gamma_{2}:=(-1,0) \times\{0\} \\
u & =0 & & \text { on } \partial \Omega \cap \partial \Omega_{j} .
\end{aligned}\right.
$$

Note that a lower bound for the essential spectrum of the eigenvalue problems on $\Omega_{2}$ and $\Omega_{3}$ is given by $\pi^{2}+1$ due to the compact support of $\bar{c}$. Using separation of variables on each of the above rectangles we can compute fundamental systems for the resulting ODE problems. Problem (32) (for $j=1,2,3$ ) thus leads to transcendental equations in $\kappa$, whose solutions are the eigenvalues of (32). By interval bisection and an Interval Newton method we can compute enclosures of these roots and therefore enclosures for all eigenvalues below $\pi^{2}+1-\varepsilon$, with some appropriately chosen $\varepsilon>0$, of the three eigenvalue problems. Let $\kappa_{1}^{(0)} \leq \kappa_{2}^{(0)} \leq \ldots \leq \kappa_{L}^{(0)}$ denote the union of all these eigenvalues (of all three problems) ordered by magnitude and counted by multiplicity. The following Lemma allows us to compare these eigenvalues with the eigenvalues below $\pi^{2}+1$ of the problem

$$
\left\{\begin{aligned}
-\Delta u+u & =\kappa(1+\bar{c}) u & & \text { in } \Omega \\
u & =0 & & \text { on } \partial \Omega
\end{aligned}\right.
$$

which we denote by $\kappa_{i}^{(\infty)}$, ordered by magnitude and counted by multiplicity.

Lemma 2. For all $i=1, \ldots, L$ we have $\kappa_{i}^{(0)} \leq \kappa_{i}^{(\infty)}$, provided that an $i$-th eigenvalue $\kappa_{i}^{(\infty)}<$ $\pi^{2}+1$ of (33) exists.

Proof. Let $V:=\left\{u \in L^{2}(\Omega):\left.u\right|_{\Omega_{j}} \in H^{1}\left(\Omega_{j}\right),\left.u\right|_{\partial \Omega_{\cap} \partial \Omega_{j}}=0\right.$ for $\left.j=1,2,3\right\}$. Since $V \supset H_{0}^{1}(\Omega)$ we have due to Poincaré's min-max principle:

$$
\begin{aligned}
\kappa_{i}^{(0)} & =\inf _{\substack{U \subset V \text { subspace } \\
\operatorname{dim} U=i}} \max _{u \in U \backslash\{0\}} \frac{\langle\nabla u, \nabla u\rangle_{L^{2}}+\langle u, u\rangle_{L^{2}}}{\langle(1+\bar{c}) u, u\rangle_{L^{2}}} \\
& \leq \inf _{\substack{U \subset H_{0}^{1}(\Omega) \text { subspace } \\
\operatorname{dim} U=i}} \max _{u \in U\{0\}} \frac{\langle\nabla u, \nabla u\rangle_{L^{2}}+\langle u, u\rangle_{L^{2}}}{\langle(1+\bar{c}) u, u\rangle_{L^{2}}}=\kappa_{i}^{(\infty)} .
\end{aligned}
$$


In principle, we can construct a homotopy connecting problems (32) and (33), but this is unnecessary, since a pure comparison of these two problems already leads to the desired rough lower bound for some higher eigenvalue of (33). More precisely, numerical Rayleigh-Ritz computations (Theorem 2) for problem (33), with some suitably chosen $n \leq L-1$, turn out to give bounds $\bar{\kappa}_{1}^{(\infty)} \leq \cdots \leq \bar{\kappa}_{n+1}^{(\infty)}<\pi^{2}+1$, whence by Theorem 2 at least $n+1$ eigenvalues $\kappa_{1}^{(\infty)} \leq \cdots \leq \kappa_{n+1}^{(\infty)}$ of problem (33) below $\pi^{2}+1$ exist, and $\kappa_{i}^{(\infty)} \leq \bar{\kappa}_{i}^{(\infty)}$. Moreover, the computations give $\bar{\kappa}_{n}^{(\infty)}<\kappa_{n+1}^{(0)}$, whence we can find some $\rho \in \mathbb{R}$ such that

$$
\bar{\kappa}_{n}^{(\infty)}<\rho \leq \kappa_{n+1}^{(0)} \stackrel{\text { Lemma } 2}{\leq} \kappa_{n+1}^{(\infty)}<\pi^{2}+1 .
$$

As a second step we have to connect problem (33), with piecewise constant coefficient function on the right-hand-side, with our original eigenvalue problem (26). For this purpose we define $c_{s}:=(1-s) \bar{c}+s(3|\omega| \omega)$ for $0 \leq s \leq 1$ and consider the $s$-dependent eigenvalue problem

$$
\int_{\Omega}[\nabla u \cdot \nabla \varphi+u \varphi] d x=\tilde{\kappa}^{(s)} \int_{\Omega}\left(1+c_{s}\right) u \varphi d x \quad \text { for all } \varphi \in H_{0}^{1}(\Omega) .
$$

Obviously, for $s=0$ this eigenvalue problem equals (33) (i.e. $\tilde{\kappa}_{i}^{(0)}=\kappa_{i}^{(\infty)}$ ) and for $s=1$ we have problem (26). Moreover, by Poincaré's min-max principle, the eigenvalues of (35) increase along the homotopy since $\bar{c} \geq 3|\omega| \omega$, i.e. for $0 \leq s \leq t \leq 1$ we have $\tilde{\kappa}_{i}^{(s)} \leq \tilde{\kappa}_{i}^{(t)}$ as long as $\tilde{\kappa}_{i}^{(t)}<\pi^{2}+1$. Step by step, this provides numbers $\rho$ (see (28), (29)) for the application of Theorem 3 to problem (35), for an increasing (finite) sequence of $s$-values. A detailed description of this homotopy method can be found e.g. in [9].

\section{$6 \quad$ Numerical Results}

\subsection{Practical computation of the residuum}

We first want to comment on some technical difficulties arising in the defect computation. Recall that we can estimate the residuum by:

$$
\left\|-\Delta \omega-|\omega|^{3}\right\|_{H^{-1}} \leq\|\nabla \omega-\tilde{\rho}\|_{L^{2}}+C_{2}\left\|-\operatorname{div} \tilde{\rho}-|\omega|^{3}\right\|_{L^{2}},
$$

where $\tilde{\rho} \in H(\operatorname{div}, \Omega)$ approximately minimizes the right-hand side of (36) and therefore is an approximation of $\nabla \omega$. The approximate solution $\omega$ is of the form $\omega=\tilde{a} w+v$, with a corner singular function $w=\lambda \gamma \in H_{0}^{1}(\Omega)$ (where $\gamma$ is given by (17) and $\lambda(x, y)=\left(1-x^{2}\right)^{2}(1-$ $\left.y^{2}\right)^{2} \chi_{(-1,1)^{2}}$ ) and a finite element approximation $v \in V_{N}$ of the regular part (compare (21); now we write $v$ instead of $\tilde{v})$. Here $V_{N}$ denotes an $H_{0}^{1}$-conforming finite element space of dimension $N$. Let now $\tilde{\rho}_{v} \in\left(V_{N}\right)^{2}$ be an approximation of $\nabla v$ (as well as an approximate solution of $\operatorname{div} \rho=-|\omega|^{3}-\tilde{a} \Delta w$; compare (20) $)$. $\tilde{\rho}_{v}$ is computed by approximate minimization of $\|\rho-\nabla v\|_{L^{2}}^{2}+C_{2}^{2}\left\|\operatorname{div} \rho+\tilde{a} \Delta w+|\omega|^{3}\right\|_{L^{2}}^{2}$ in $\left(V_{N}\right)^{2}$. Define

$$
\tilde{\rho}:=\tilde{a} \nabla w+\tilde{\rho}_{v} .
$$

Substituting the expressions for $\omega$ and $\tilde{\rho}$ in (36) yields

$$
\left\|-\Delta \omega-|\omega|^{3}\right\|_{H^{-1}} \leq\left\|\nabla v-\tilde{\rho}_{v}\right\|_{L^{2}}+C_{2}\left\|-\tilde{a} \Delta w-\operatorname{div} \tilde{\rho}_{v}-|\tilde{a} w+v|^{3}\right\|_{L^{2}} .
$$


The first summand on the right-hand side of (37) can be computed exactly using a quadrature rule of sufficiently high degree together with interval arithmetic, as $\tilde{\rho}$ is an element of $\left(V_{N}\right)^{2}$ and also $\nabla v$ is piecewise polynomial in each component. Due to the mixture of cartesian and polar coordinates in the second summand, a verified computation of a tight upper bound for this term is technically non-trivial. We interpolate the singular function $\gamma(r, \varphi)$, as well as $x \frac{\partial \gamma}{\partial x}(r, \varphi)=$ $-\frac{2}{3} r^{2 / 3} \cos \varphi \sin \frac{\varphi}{3}$ and $y \frac{\partial \gamma}{\partial y}(r, \varphi)=\frac{2}{3} r^{2 / 3} \sin \varphi \cos \frac{\varphi}{3}$ in the finite element space $V_{N}$, and replace the corresponding terms in the second summand in (37) by these interpolations. Now the integrand is piecewise polynomial, and hence the integral can be computed exactly. The remaining task is to bound the interpolation errors, which after some elementary estimations amounts to the computation of bounds to

$$
\int_{K}(\gamma-I(\gamma))^{2} d(x, y)
$$

for each element $K$ (with $I(\gamma)$ denoting the interpolation), and to analogous terms for $x \frac{\partial \gamma}{\partial x}$ and $y \frac{\partial \gamma}{\partial y}$. For this purpose we cover each element $K$ by a finite union of circular segments (i.e. rectangles in polar coordinates), replace $K$ in (38) by this union (giving an upper bound to the integral), and transform the integral to polar coordinate integration (over a union of rectangles), which can be carried out in closed form, using Maple for calculating primitive functions.

\subsection{Computational Results}

We will now report on some of the numerical results that finally prove the existence of a solution of problem (5) together with an error bound. All computations have been carried out on the parallel cluster OTTO of the Institute for Applied and Numerical Mathematics at Karlsruhe Institute of Technology. We used the finite element software $\mathrm{M}++$ [24, which is written in $\mathrm{C}++$. For interval arithmetic we used the libraries C-XSC [16 as well as MPFR and MPFI [8]. Our source code is available on http://www.math.kit.edu/user/mi1/Plum/PaperPPR/ or upon request to the third author.

As already mentioned in section 3, we used the computational domain $\Omega_{0}=\Omega \cap(-3,3)^{2}$, and taking symmetry into account we restricted ourselves to the half domain $\tilde{\Omega}_{0}=\operatorname{conv}\{(0,0),(3,0)$, $(3,1),(-1,1)\}$, imposing Neumann boundary conditions on $\partial \tilde{\Omega}_{0} \backslash \partial \Omega_{0}=: \Gamma_{N}$. This diminishes the constant $K$ in (7), since only eigenfunctions which are symmetric w.r.t. $\Gamma_{N}$ have to be considered in the eigenvalue problem (26), and hence less eigenvalues contribute to the minimum in (23). Furthermore, symmetry reduces the computational effort. Using the space $\left\{u \in H^{1}\left(\tilde{\Omega}_{0}\right): u=0\right.$ on $\left.\partial \Omega_{0} \cap \partial \tilde{\Omega}_{0}\right\}$ in all computations has lead to an approximate solution that is symmetric w.r.t. $\Gamma_{N}$ and eventually - after a successful application of our theoretical results - to a symmetric solution $u$ of (5).

In our computations we used Serendipity finite elements, whose nodes are given by corners and midpoints of the elements. Figure 1 shows the computed approximate solution $\omega_{0} \in H_{0}^{1}\left(\Omega_{0}\right)$ (see (16)) on the full domain $\Omega_{0}$.

To compute bounds for eigenvalues of (26) we used, as described before, a domain decomposition method to obtain lower bounds for the base eigenvalues and a homotopy method to 


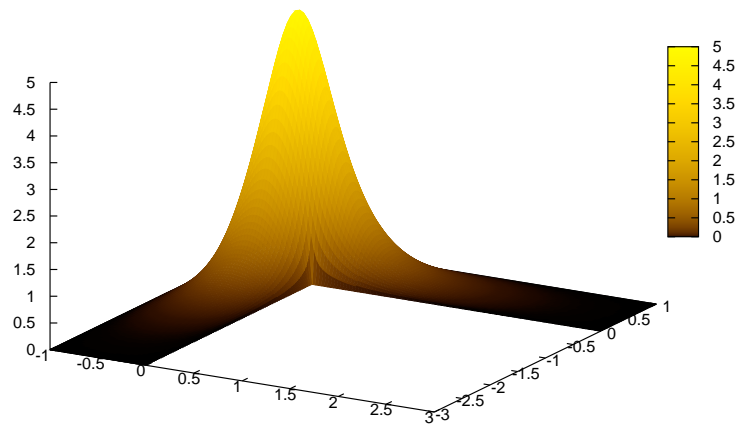

Figure 1: Approximate solution $\omega_{0} \in H_{0}^{1}\left(\Omega_{0}\right)$

connect the base problem (33) with our original eigenvalue problem (26). We note that for the eigenvalue computation we did not use the approximation $\omega=\tilde{a} w+v$, but an interpolation $\omega_{F E}:=I_{V_{\tilde{N}}}(\omega)$ in a finite element space $V_{\tilde{N}}$ which is coarser than $V_{N}$. This avoids complicated integration during the homotopies and saves computation time. Eventually we obtain a bound for the inverse of the linearization at $\omega_{F E}$, which can then be used to compute the corresponding bound for $L_{\omega}$ by Lemma 1 (b), with $\tilde{u}=\omega_{F E}$ and $u=\omega$.

Recall that the base problem is given by

$$
\left\{\begin{aligned}
-\Delta u+u & =\kappa(1+\bar{c}) u & & \text { in } \Omega \\
u & =0 & & \text { on } \partial \Omega,
\end{aligned}\right.
$$

where now $\bar{c} \in L^{\infty}(\Omega)$ is chosen such that $\bar{c} \geq 3\left|\omega_{F E}\right| \omega_{F E}$ in $\Omega$ and is constant on the rectangles $(-1,0) \times(0,1),(0,1) \times(0,1),(1,3) \times(0,1)$ and $(3, \infty) \times(0,1)$ as well as on $(-1,0) \times(-1,0),(-1,0) \times(-3,-1)$ and $(-1,0) \times(-\infty,-3)$. This choice defines also the aforementioned comparison problem for the domain decomposition.

In the following table and figure we display some results of our eigenvalue computations. The first column of the table shows lower bounds $\underline{\kappa}_{i}^{(0)}$ for the eigenvalues $\kappa_{i}^{(0)}$ of the comparison problem, which by Lemma 2 constitute lower bounds (indexwise) for the eigenvalues $\kappa_{i}^{(\infty)}=\tilde{\kappa}_{i}^{(0)}$ of the base eigenvalue problem (39). The second column of the table shows upper bounds $\bar{\kappa}_{i}^{(\infty)}$ for the eigenvalues $\kappa_{i}^{(\infty)}$, computed by Theorem 2. In particular $\bar{\kappa}_{11}^{(\infty)}<\underline{\kappa}_{12}^{(0)}\left(\leq \kappa_{12}^{(\infty)}\right)$, whence (34) holds for $n=11$ and $\rho=\underline{\kappa}_{12}^{(0)}$, which enables the start of the homotopy for problem (35). The figure shows the course of the homotopy, where we started with the a-priori lower bound $\underline{\kappa}_{12}^{(0)}$ for the 12 th eigenvalue $\tilde{\kappa}_{12}^{(0)}=\kappa_{12}^{(\infty)}$ of problem (35) with $s=0$ (and $\omega$ replaced by $\left.\omega_{F E}\right)$. For additional illustration, the figure also contains approximations to the eigenvalues $\tilde{\kappa}_{1}^{(0)}, \ldots, \tilde{\kappa}_{12}^{(0)}$. At the end of the homotopy we obtained a lower bound for the third eigenvalue of problem (26) (with $\omega$ replaced by $\omega_{F E}$ ). Using the Lehmann-Goerisch Theorem once again we computed the desired lower bound for the second eigenvalue of (26) (with $\omega_{F E}$ instead of $\omega$ ), which is the smallest eigenvalue above 1 . Finally an application of the Rayleigh-Ritz method yields an upper bound for the first eigenvalue of (26) (with $\omega$ replaced by $\omega_{F E}$ ) and Lemma 1 (b) then gives a bound for the inverse of the linearization at $\omega$. 


\begin{tabular}{|c|c|c|}
\hline$i$ & $\underline{\kappa}_{i}^{(0)}$ & $\bar{\kappa}_{i}^{(\infty)}$ \\
\hline \hline 1 & 0.08291 & 0.18117 \\
\hline 2 & 0.35867 & 0.48073 \\
\hline 3 & 0.52231 & 0.69818 \\
\hline 4 & 0.63443 & 0.84524 \\
\hline 5 & 0.91020 & 1.02708 \\
\hline 6 & 1.08005 & 1.43595 \\
\hline 7 & 1.18596 & 1.52509 \\
\hline 8 & 1.73749 & 1.93536 \\
\hline 9 & 1.73749 & 1.97598 \\
\hline 10 & 1.79188 & 2.07092 \\
\hline 11 & 2.01325 & 2.27739 \\
\hline 12 & 2.35653 & 2.75040 \\
\hline
\end{tabular}

Table 1: Eigenvalues of the comparison problem and the base problem

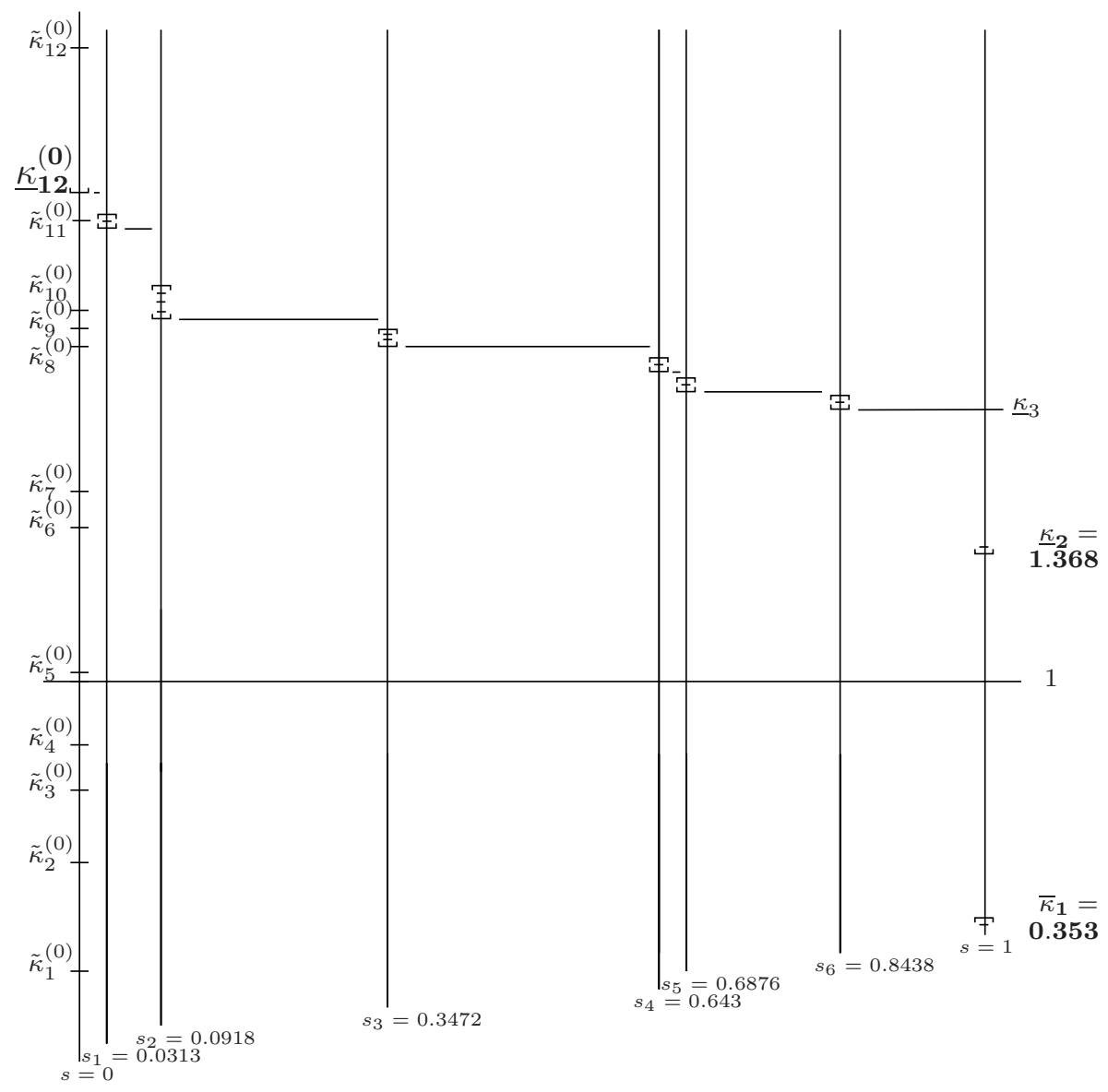

Figure 2: Course of the homotopy

An embedding constant $C_{4}$ for the embedding $H_{0}^{1}(\Omega) \hookrightarrow L^{4}(\Omega)$, which is needed for the application of Theorem 1, can be computed using Lemma 2 in [20]. The computation requires a lower bound for the smallest Dirichlet eigenvalue of $-\Delta$ on $\Omega$, which can again be obtained using the Lehmann-Goerisch method.

Verified bounds for some of the relevant data are given by:

$$
\begin{aligned}
\|\omega\|_{L^{4}(\Omega)} & \leq 3.014333 \\
\left\|-\Delta \omega-|\omega|^{3}\right\|_{H^{-1}(\Omega)} & \leq 0.001699 \\
K^{\mathrm{sym}} & =3.722884 \\
C_{4} & =0.462000 \\
\alpha & =0.006471
\end{aligned}
$$

where $K^{\text {sym }}$ denotes a constant satisfying

$$
\|v\|_{H_{0}^{1}(\Omega)} \leq K^{\mathrm{sym}}\left\|L_{\omega}[v]\right\|_{H^{-1}(\Omega)} \quad \text { for all } v \in H_{0}^{1}(\Omega) \text { which are symmetric w.r.t. } \Gamma_{N} \text {. }
$$

Thus, the existence of a symmetric solution $u \in H_{0}^{1}(\Omega)$ to problem (5) with $\|u-\omega\|_{H_{0}^{1}} \leq$ 0.006471 is proved. 


\section{$7 \quad$ Nondegeneracy of the solution}

Besides decay properties, which we will analyze in the next section, we also prove nondegeneracy of the solution $u \in H_{0}^{1}(\Omega)$ we have obtained, i.e. that 0 is not in the spectrum of the linearization $L_{u}$ at $u$. For this purpose we note that due to the restriction to the half domain $\tilde{\Omega}_{0}$ with Neumann boundary conditions on $\Gamma_{N}$, the eigenvalue bounds computed in the previous section are not sufficient to prove nondegeneracy of $u$ in the whole space $H_{0}^{1}(\Omega)$. Indeed, we have to compute also eigenvalues of $\Phi^{-1} L_{\omega}: H_{0}^{1}(\Omega) \rightarrow H_{0}^{1}(\Omega)$ corresponding to antisymmetric eigenfunctions. This can be done using the same methods as described in the previous sections, this time imposing Dirichlet boundary conditions on $\Gamma_{N}$. Altogether, bounds for the smallest eigenvalues of $\Phi^{-1} L_{\omega}$ are given by

$$
\nu_{1} \leq-1.8359, \quad \nu_{2} \geq 0.1116
$$

where the first eigenvalue corresponds to a symmetric eigenfunction, while the second one corresponds to an antisymmetric one. Thus, 0 is not in the spectrum of $\Phi^{-1} L_{\omega}$. To obtain the corresponding result also for $\Phi^{-1} L_{u}$, we first apply Lemma 1 (b), with $p_{1}=p_{2}=p_{3}=p_{4}=4$, to obtain

Corollary 1. Let $u, \tilde{u} \in H_{0}^{1}(\Omega)$ and let $\operatorname{dist}\left(\sigma\left(\Phi^{-1} L_{\tilde{u}}\right), 0\right)>0$. Furthermore assume that, for some $\alpha>0$,

$$
\|u-\tilde{u}\|_{H_{0}^{1}} \leq \alpha .
$$

If

$$
\kappa:=3 C_{4}^{3}\left(C_{4} \alpha+2\|\tilde{u}\|_{L^{4}}\right) \alpha<\operatorname{dist}\left(\sigma\left(\Phi^{-1} L_{\tilde{u}}\right), 0\right)
$$

then nondegeneracy of $u$ follows.

For the proof note that $\operatorname{dist}\left(\sigma\left(\Phi^{-1} L_{\tilde{u}}\right), 0\right)>0$ implies $\|v\|_{H_{0}^{1}} \leq K\left\|L_{\tilde{u}}[v]\right\|_{H^{-1}}\left(v \in H_{0}^{1}(\Omega)\right)$ with $K=\frac{1}{\operatorname{dist}\left(\sigma\left(\Phi^{-1} L_{\tilde{u}}\right), 0\right)}$ (see also (22),$\left.(\underline{23})\right)$.

We apply Corollary 1 with $\tilde{u}=\omega$, and $u$ being the solution of (15) given by Theorem 11, and thus satisfying

$$
\|u-\omega\|_{H_{0}^{1}} \leq \alpha
$$

Using the data of the previous paragraph we obtain $\kappa=0.01473$ and therefore, by (41) and (42), nondegeneracy of $u$. Moreover, using Poincaré's min-max principle, we can deduce from (41) and (43) that the Morse index of $u$ is 1 ; we omit the details here.

\section{Decay at infinity}

We finally prove that the solution $u \in H_{0}^{1}(\Omega)$ decays exponentially at infinity.

Theorem 4. Let $\phi(z)=\sin (\pi z)$. There exists a constant $\beta>0$ such that for $x \rightarrow \infty$ we have

$$
\begin{aligned}
u(x, y) & =\beta e^{-\pi x} \phi(y)+o\left(e^{-\pi x} \phi(y)\right) \\
\frac{\partial u}{\partial x}(x, y) & =-\beta \pi e^{-\pi x} \phi(y)+o\left(e^{-\pi x} \phi(y)\right)
\end{aligned}
$$


and analogously for $y \rightarrow-\infty$

$$
\begin{aligned}
u(x, y) & =\beta e^{\pi y} \phi(x+1)+o\left(e^{\pi y} \phi(x+1)\right) \\
\frac{\partial u}{\partial y}(x, y) & =\beta \pi e^{\pi y} \phi(x+1)+o\left(e^{\pi y} \phi(x+1)\right) .
\end{aligned}
$$

Proof. For the proof it is sufficient to show that $u \in C\left(\bar{\Omega} \backslash[-2.5,2.5]^{2}\right)$ and $u(x, y) \rightarrow 0$ as $x \rightarrow \infty$ uniformly in $y$ (and similarly $u(x, y) \rightarrow 0$ as $y \rightarrow-\infty$ uniformly in $x$ ). Then Prop. 4.2. in [7] can be applied to the half strips $\Omega \cap\{x>2.5\}$ and $\Omega \cap\{y<-2.5\}$, and implies the theorem.

So let $r=\frac{1}{\sqrt{2}}+\varepsilon$ (for some sufficiently small $\varepsilon>0$ ) and let $\Omega_{c} \subset \Omega$ be a $C^{2}$-domain such that $\{(x, y) \in \Omega: 2.5<x<3.5\} \subset \Omega_{c} \subset\{(x, y) \in \Omega: 2<x<4\}$ and $\operatorname{dist}\left(\Omega_{c} \cap B_{r}\left(3, \frac{1}{2}\right),\left(\partial \Omega_{c}\right) \cap \Omega\right)>0$. Choose moreover a cutoff-function $\zeta \in C^{\infty}\left(\bar{\Omega}_{c}\right)$ such that $\zeta=0$ on $\left(\partial \Omega_{c}\right) \cap \Omega$ and $\zeta=1$ in $\Omega_{c} \cap B_{r}\left(3, \frac{1}{2}\right)$.

Define now $\tilde{u}=u \zeta \in H_{0}^{1}\left(\Omega_{c}\right)$; then for every $\varphi \in H_{0}^{1}\left(\Omega_{c}\right)$ we have, since $\int_{\Omega_{c}} \nabla u \cdot \nabla(\zeta \varphi) d x=$ $\int_{\Omega_{c}} u^{3} \zeta \varphi d x$

$$
\begin{aligned}
\int_{\Omega_{c}} \nabla \tilde{u} \cdot \nabla \varphi d x & =\int_{\Omega_{c}}(\zeta \nabla u \cdot \nabla \varphi+u \nabla \zeta \cdot \nabla \varphi) d x \\
& =\int_{\Omega_{c}} \nabla u \cdot \nabla(\zeta \varphi) d x-\int_{\Omega_{c}}(2 \nabla u \cdot \nabla \zeta+u \Delta \zeta) \varphi d x=\int_{\Omega_{c}} f \varphi d x,
\end{aligned}
$$

where $f:=u^{3} \zeta-2 \nabla u \cdot \nabla \zeta-u \Delta \zeta \in L^{2}\left(\Omega_{c}\right)$.

Then Theorem 9.8 in [3] yields (with $\Omega_{1}:=\Omega_{c} \cap B_{r}\left(3, \frac{1}{2}\right)$ ):

$$
\tilde{u} \in H^{2}\left(\Omega_{1}\right) \quad \text { and } \quad\|\tilde{u}\|_{H^{2}\left(\Omega_{1}\right)} \leq \gamma\left(\|\tilde{u}\|_{L^{2}\left(\Omega_{c}\right)}+\|f\|_{L^{2}\left(\Omega_{c}\right)}\right)
$$

for some constant $\gamma$ not depending on $u$.

Since $\tilde{u}=u$ in $\Omega_{1}, \zeta \in C^{\infty}\left(\bar{\Omega}_{c}\right)$ and $\|f\|_{L^{2}\left(\Omega_{c}\right)} \leq C\|u\|_{H^{1}\left(\Omega_{c}\right)}$, we obtain

$$
u \in H^{2}\left(\Omega_{1}\right) \text { and }\|u\|_{H^{2}\left(\Omega_{1}\right)} \leq \widehat{C}\|u\|_{H^{1}\left(\Omega_{c}\right)} .
$$

Define now

$$
u_{n}(x, y):=u(x+n, y), \quad n \in \mathbb{N},(x, y) \in \Omega_{c} .
$$

Then, since $\int_{\Omega_{c}} \nabla u_{n} \cdot \nabla \varphi d x=\int_{\Omega_{c}} u_{n}^{3} \varphi d x$ for $\varphi \in H_{0}^{1}\left(\Omega_{c}\right)$, by the above we obtain

$$
u_{n} \in H^{2}\left(\Omega_{1}\right) \quad \text { and } \quad\left\|u_{n}\right\|_{H^{2}\left(\Omega_{1}\right)} \leq \widehat{C}\left\|u_{n}\right\|_{H^{1}\left(\Omega_{c}\right)} .
$$

Using the embedding $H^{2}\left(\Omega_{1}\right) \hookrightarrow C\left(\bar{\Omega}_{1}\right)$ implies (with $K$ denoting the embedding constant):

$$
u_{n} \in C\left(\bar{\Omega}_{1}\right) \quad \text { and } \quad\left\|u_{n}\right\|_{C\left(\bar{\Omega}_{1}\right)} \leq K \widehat{C}\left\|u_{n}\right\|_{H^{1}\left(\Omega_{c}\right)} .
$$

Therefore we have $u \in C(\bar{\Omega} \cap\{x>2.5\})$. Moreover, $\left\|u_{n}\right\|_{H^{1}\left(\Omega_{c}\right)}=\|u\|_{H^{1}\left(\Omega_{c}+(n, 0)\right)} \rightarrow 0$ $(n \rightarrow \infty)$ since $u \in H^{1}(\Omega)$, whence $\left\|u_{n}\right\|_{C\left(\bar{\Omega}_{1}\right)} \rightarrow 0$ as $n \rightarrow \infty$ follows, implying $u \rightarrow 0$ as $x \rightarrow \infty$ uniformly in $y$. 


\section{References}

[1] N. Ackermann, M. Clapp, And F. PaCella, Self-focusing multibump standing waves in expanding waveguides, Milan J. Math., 79 (2011), pp. 221-232.

[2] —_, Alternating sign multibump solutions of nonlinear elliptic equations in expanding tubular domains, Comm. Partial Differential Equations, 38 (2013), pp. 751-779.

[3] S. Agmon, Lectures on Elliptic Boundary Value Problems, no. 2 in Van Nostrand Mathematical Studies, 1965.

[4] T. Bartsch, M. Clapp, M. Grossi, and F. Pacella, Asymptotically radial solutions in expanding annular domains, Math. Ann., 352 (2012), pp. 485-515.

[5] H. BehnKe AND F. GoERISCH, Inclusions for eigenvalues of selfadjoint problems, in Topics in Validated Computations, J. Herzberger, ed., Elsevier, 1994, pp. 277-322.

[6] H. Behnke, U. Mertins, M. Plum, And C. Wieners, Eigenvalue Inclusions via Domain Decomposition, Proc. R. Soc. Lond. A (2000), 456, pp. 2717-2730.

[7] H. Berestycki and L. Nirenberg, Some Qualitative Properties of Solutions of Semilinear Elliptic Equations in Cylindrical Domains, in Analysis, et cetera, P. H. Rabinowitz, ed., Academic Press, 1990, pp. 115-164.

[8] F. Blomquist, W. Hofschuster, And W. Krämer, C-XSC-Langzahlarithmetiken für reelle und komplexe Intervalle basierend auf den Bibliotheken MPFR und MPFI, Preprint BUW-WRSWT, Universität Wuppertal, 2011/1.

[9] B. Breuer, J. Horák, P. J. McKenna, And M. Plum, A computer-assisted existence and multiplicity proof for travelling waves in a nonlinearly supported beam, Journal of Differential Equations, 224 (2006), pp. 60-97.

[10] F. Catrina And Z.-Q. Wang, Nonlinear elliptic equations on expanding symmetric domains, J. Differential Equations, 156 (1999), pp. 153-181.

[11] E. N. DANCER AND S. YAN, Multibump solutions for an elliptic problem in expanding domains, Comm. Partial Differential Equations, 27 (2002), pp. 23-55.

[12] P. Grisvard, Elliptic Problems in Nonsmooth Domains, no. 24 in Monographs and Studiens in Mathematics, Pitman, 1985.

[13] — Singularities in Boundary Value Problems, no. 22 in Research Notes in Applied Mathematics, Masson, Springer-Verlag, 1992.

[14] V. Hoang, M. Plum, and C. Wieners, A computer-assisted proof for photonic band gaps, Zeitschrift für angewandte Mathematik und Physik, 60 (2009), pp. 1035-1052.

[15] T. Kato, Perturbation Theory for Linear Operators, vol. 132 of Grundlehren der mathematischen Wissenschaften, Springer-Verlag, 1976.

[16] R. Klatte, U. Kulisch, A. Wiethoff, C. Lawo, and M. Rauch, C-XSC: A $C++$ class library for extended scientific computing, Springer, 1993. 
[17] Y. Y. Li, Existence of many positive solutions of semilinear elliptic equations on annulus, J. Differential Equations, 83 (1990), pp. 348-367.

[18] P. J. McKenna, F. Pacella, M. Plum, And D. Roth, A Uniqueness Result for a Semilinear Elliptic Problem: A Computer-assisted Proof, Journal of Differential Equations, 247 (2009), pp. 2140-2162.

[19] P. J. McKenna, F. Pacella, M. Plum, and D. Roth, A computer-assisted uniqueness proof for a semilinear elliptic boundary value problem, in Inequalities and applications 2010, vol. 161 of Internat. Ser. Numer. Math., Birkhäuser/Springer, Basel, 2012, pp. 3152 .

[20] M. Plum, Existence and Multiplicity Proofs for Semilinear Elliptic Boundary Value Problems by Computer Assistance, Jahresbericht der DMV, 110 (2008), pp. 19-54.

[21] S. M. Rump, INTLAB - INTerval LABoratory, in Developments in Reliable Computing, T. Csendes, ed., Kluwer Academic Publishers, Dordrecht, 1999, pp. 77-104. http://www.ti3.tuhh.de/rump/.

[22] C. Sulem And P.-L. Sulem, The nonlinear Schrödinger equation, vol. 139 of Applied Mathematical Sciences, Springer-Verlag, New York, 1999. Self-focusing and wave collapse.

[23] T. Suzuki, Positive solutions for semilinear elliptic equations on expanding annuli: mountain pass approach, Funkcial. Ekvac., 39 (1996), pp. 143-164.

[24] C. WiEnERs, A geometric data structure for parallel finite elements and the application to multigrid methods with block smoothing, Comput. Vis. Sci., 13 (2010), pp. 161-175. 